\title{
Determination of oleic acid in the samples of sunflower seeds by method of nir-spectroscopy
}

\author{
Inna Hutsalo, Valeriy Mank, Svitlana Kovaleva
}

\author{
National University of food Technologies, Kyiv, Ukraine
}

Keywords:

Spectroscopy

Oleic

Acid

Sunflower

Seed

\section{Article history:}

Received 19.11.2016

Received in revised

form 19.03.2017

Accepted 30.03.2017

\section{Corresponding \\ author:}

Svitlana Kovaleva

E-mail:

Sval_kov@ukr.net

DOI: $10.24263 / 2304-$

974X-2017-6-1-6

\section{Abstract}

Introduction. The possibility of using NIR spectroscopy to determine oleic acid in sunflower seeds has not been studied, so research on this field is perspective.

Materials and methods. The spectra of seed samples of various sunflower varieties with a known content of oleic acid and the same samples additionally enriched with oleic acid were investigated by NIR diffusion reflectance spectroscopy with using the instrument "Infrapid-61". To process the results obtained, the methods of mathematical analysis were applied.

Results and Discussion. In the NIR spectra of samples of dried sunflower seeds, in comparison with the spectra of raw seeds, the expected decrease in the coefficient of diffuse reflection is observed in the range 1920-1940 nm related to the moisture content in the sample. Analysis of NIR spectra of a calibration series of dried seeds enriched with oleic acid shows an increase in the coefficient of diffuse reflection in the wavelength ranges $1920-1940 \mathrm{~nm}$ and $2140-2160 \mathrm{~nm}$ in proportion to the growth of the mass portion of oleic acid. Corresponding calculations, calibration curves and the obtained equation describing the dependence demonstrate a linear dependence of the reflection coefficient on the mass portion of oleic acid in the sample at the wavelength of 2140 $\mathrm{nm}$ with a confidence level of $98 \%$. The dependency found can be used for the quantitative determination of oleic acid in a sunflower seed sample of unknown composition. By the magnitude of the coefficient of diffuse reflection of the sunflower seed sample containing unknown amount of oleic acid, its mass portion in the sample can be determined from the graph. The diffuse reflectance spectra of husked and crushed seed samples and corresponding spectra of crushed sunflower seeds with husks containing the same oleate amount are practically identical with the wavelength range $1330-2370 \mathrm{~nm}$. Therefore, this method can be used for the analysis both intact seeds and seeds separated from husks. The express method of diffuse reflection of NIR spectroscopy can be considered as an alternative to chemical methods for determining the quality indicators of fatcontaining raw materials.

Conclusions. The method of NIR spectroscopy is perspective for the determining of other fatty carboxylic acids in sunflower seeds. 


\section{Introduction}

Near-infrared spectroscopy has been known as a powerful tool for analysis of chemical and physical properties without sample preparation, and it has been applied for the analysis of quality characteristics of complex multi component systems [1]. More then decade, studies on the use of NIR diffuse reflection spectroscopy have been widely carried out to analyze food products and raw materials for their production. Despite the presence of a large number of such data, sufficiently accurate mathematical models, which unambiguously correlate the absorption bands in the NIR region of the spectrum with the quantitative and sometimes qualitative composition of the samples of products under study, have been lacking [2]. The interpretation of the spectral data by the different authors' reports is different, so there are no valid reliable characteristics that could be relied on.

The possibility of using spectroscopy for the quantitative determination of oleic acid in sunflower seeds has not been studied yet, although the NIRS has been successfully used to determine diverse compounds in numerous foods and industrial crops such as sesame, soybean, perilla and peanut, sunflower, rice, maize, and sweet potato [3]. The content of oleic acid in the form of glycerol esters (oleates) in different varieties of sunflower varies from 20 to $90 \%$ and determines the nutritional value of sunflower oil [4]. Modern methods for quantitative analysis of free carboxylic acids in fats and oils are based on chemical transformations, are relatively long, conducted in special laboratories and require the use of chemical reagents. .The purpose of these studies was the development of an express method for the quantitative determination of oleic acid in samples of fatcontaining raw materials on the basis of diffuse reflection spectroscopy in the near IR region [5].

\section{Materials and methods}

\section{Seed samples of sunflower}

Different varieties of sunflower seed were obtained from the Institute of Oilseeds (Zaporizhia). The content of oleic acid in seeds was determined in the factory laboratory. All seed samples were stored at $+10^{\circ} \mathrm{C}$ prior to analysis. Standard oleic acid was obtained from the University of Food Technologies [6].

\section{Sample preparation}

To carry out the experiment under conditions close to the conveyor regime, the samples were subjected to minimal processing. Two samples of raw sunflower seeds of each variety were taken. One sample of each pair was ground with the husks, the seeds of another sample were separated from the husks and also ground. To ensure the same level of grinding and eliminate the influence of the particle size factor on the resulting spectra, the crushed samples are screened through a sieve with hole diameter of $1 \mathrm{~mm}$. To reduce the influence of moisture on the spectral characteristics of the samples, the crushed seeds without the husks were dried to a constant mass, using an infrared dryer.

\section{Methods}

By the method of investigation, a method of diffuse reflection in the near region of the infrared spectrum was chosen for the determination of oleic acid in samples of sunflower oil. The essence of the method consists in comparing the characteristics of the infrared 
spectra of the sample that is under investigation, with the spectra of the preliminary investigated calibration series of samples. Infrared spectra of diffuse reflection for samples of sunflower seeds were recorded using the instrument "Infrapid-61" in the wavelength region $1330-2370 \mathrm{~nm}$.

\section{Data processing}

The equations for NIRS prediction, calibration coefficients and statistical parameters characterizing the calibration of the instrument were developed with the regression method.

\section{Preparation of the calibration series of raw material samples}

The use of diffuse reflection spectroscopy involves conducting a procedure for preliminary calibration of the instrument using standard methods [7]. Samples for the calibration series were prepared by adding free oleic acid to the ground seeds characterized with known content of oleates [8]. For this purpose, a solution of oleic acid in a volatile organic solvent was evenly sprayed over the surface of the sample stirring it. The increase in the mass fraction of oleic acid was controlled by measuring the mass of the sample after a short drying term at a temperature of $35^{\circ} \mathrm{C}$.

\section{Spectra collection and pretreatment}

Prepared samples of ground seeds were loaded into the cuvette compartment of the instrument and their spectra were recorded. The procedure for analyzing all samples involves recording the spectrum of the standard that is in the instrument, recording the spectrum of the prepared samples that are under the study, and processing the obtained results with the appropriate software [8]. The time for recording of the spectrum of one sample does not exceed 2 minute. The reflectance data were recorded as a reflectance coefficient $\mathrm{R}$ at $10 \mathrm{~nm}$ intervals, and 3 scans were averaged for each sample.

\section{Results and discussion}

The results of computer processing of the obtained spectra of samples of crushed seeds with husks containing oleic acid from 79 to $90 \%$ are shown in Figure 1 . Comparative analysis of spectra of samples series of crushed seeds demonstrates their similarity in that wave region (from 1370 to $2370 \mathrm{~nm}$ ) [9]. Spectra of samples of crushed seeds without husks with the corresponding content of oleic esters are shown in Figure 2 and they also seem to be similar. The experiments show that the method is suitable for the analysis both intact seeds and seeds separated from husks [10].

To compare the spectra of diffuse reflection of seed samples with husks and without husks, two sunflower varieties, exactly "Smak" and "KP1 1B" containing high (73.6\%) and low $(29.78 \%)$ amount of oleates, were investigated. The results are shown in Figure 3 .

As a result of the analysis of these IR spectra, no significant differences were found in the NIR region. Spectra of dried seed samples are shown in Figure 4. 


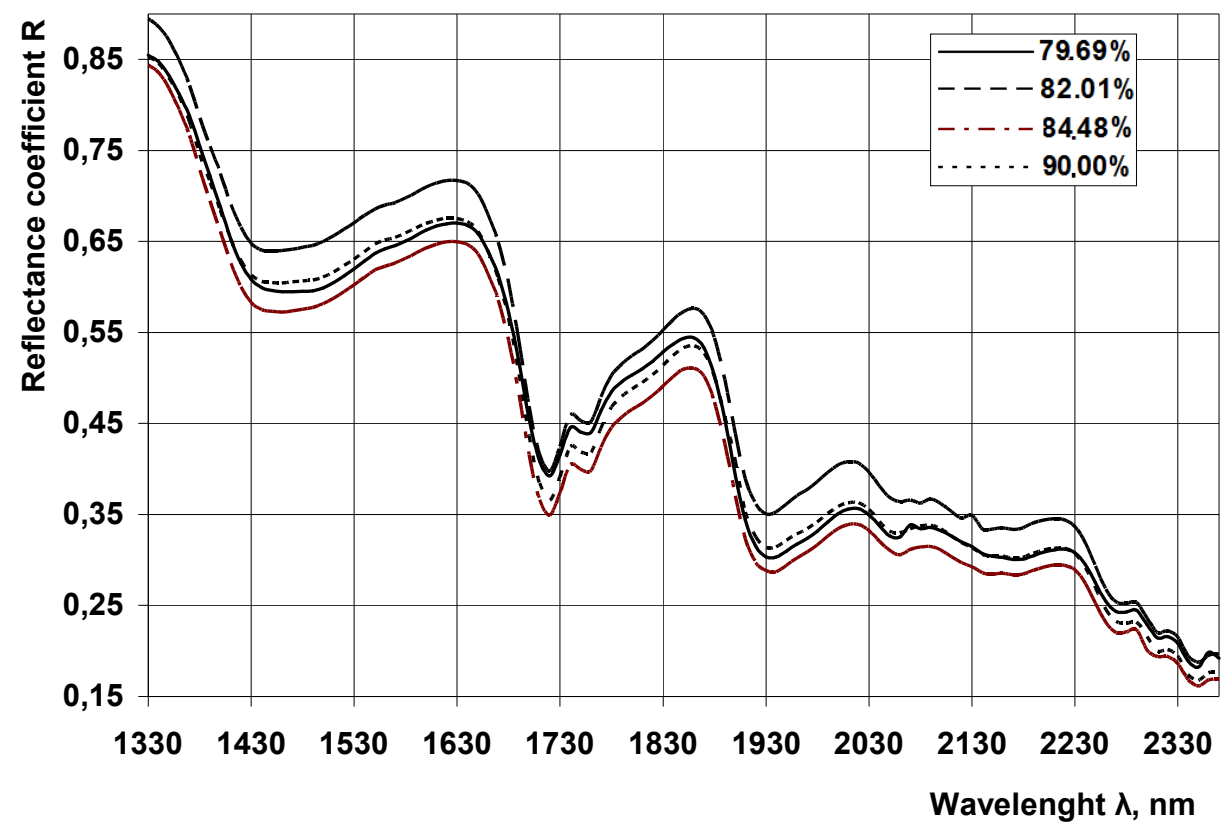

Figure 1. Diffuse reflection NIR spectra of samples of crushed sunflower seeds with husks containing oleic acid from 79 to $90 \%$.

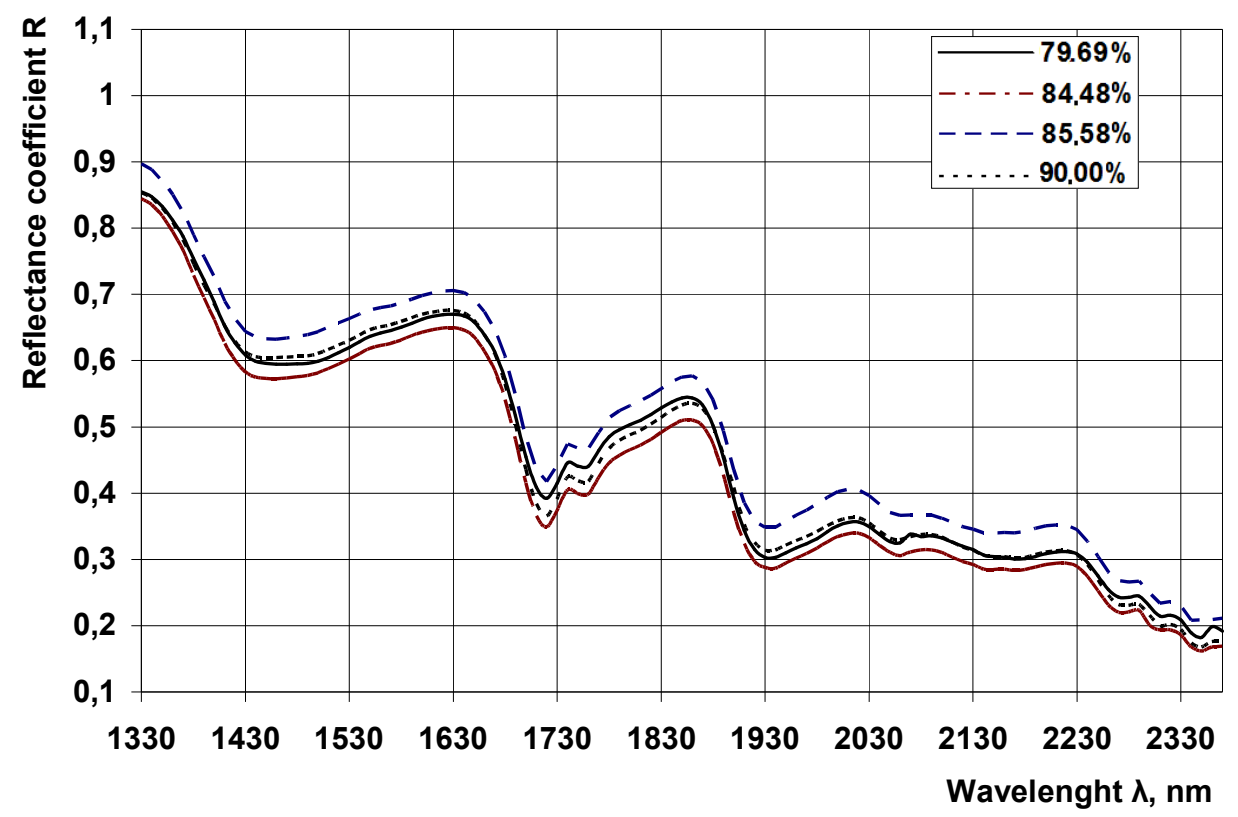

Figure 2. Diffuse reflection NIR spectra of samples of crushed sunflower seeds without husks containing oleic acid from 79 to $90 \%$. 


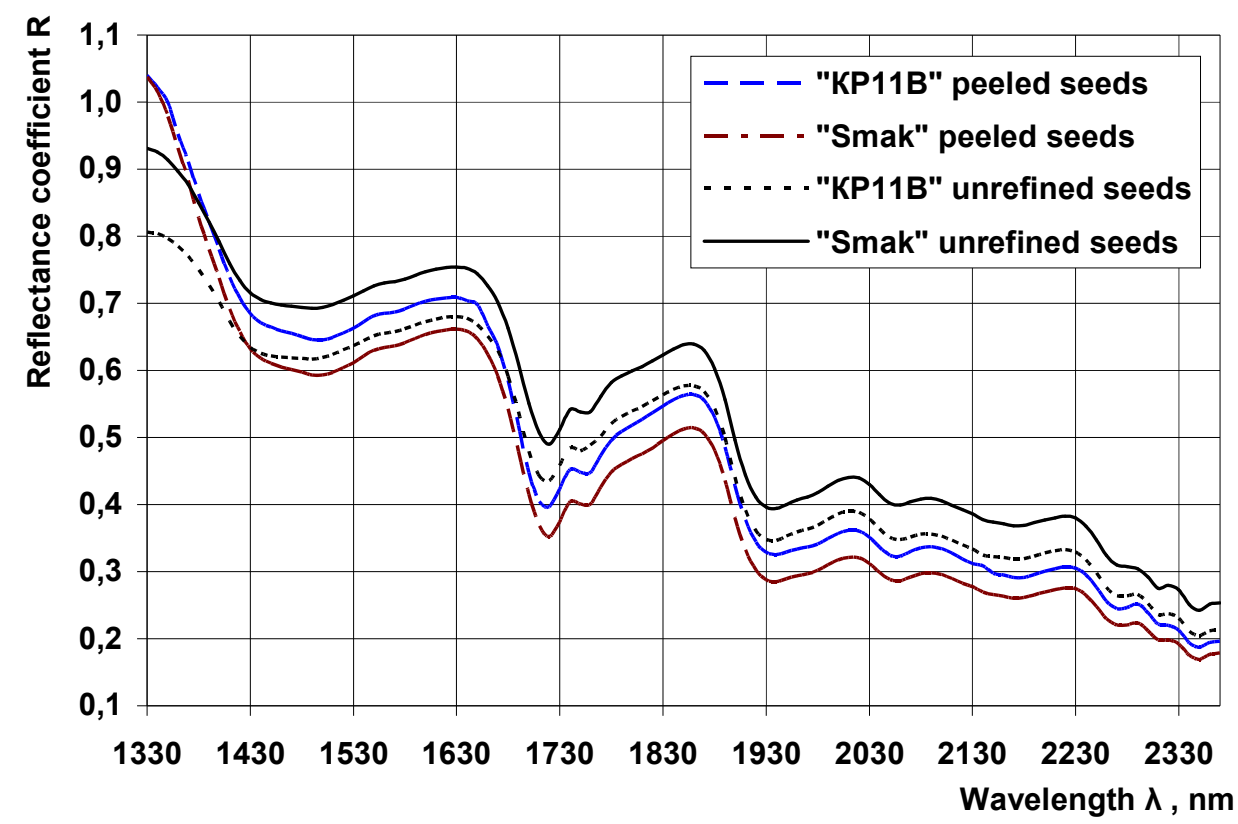

Figure 3. Diffuse reflection NIR spectra of samples of sunflower seed varieties "Smak" and "KP1 1B"

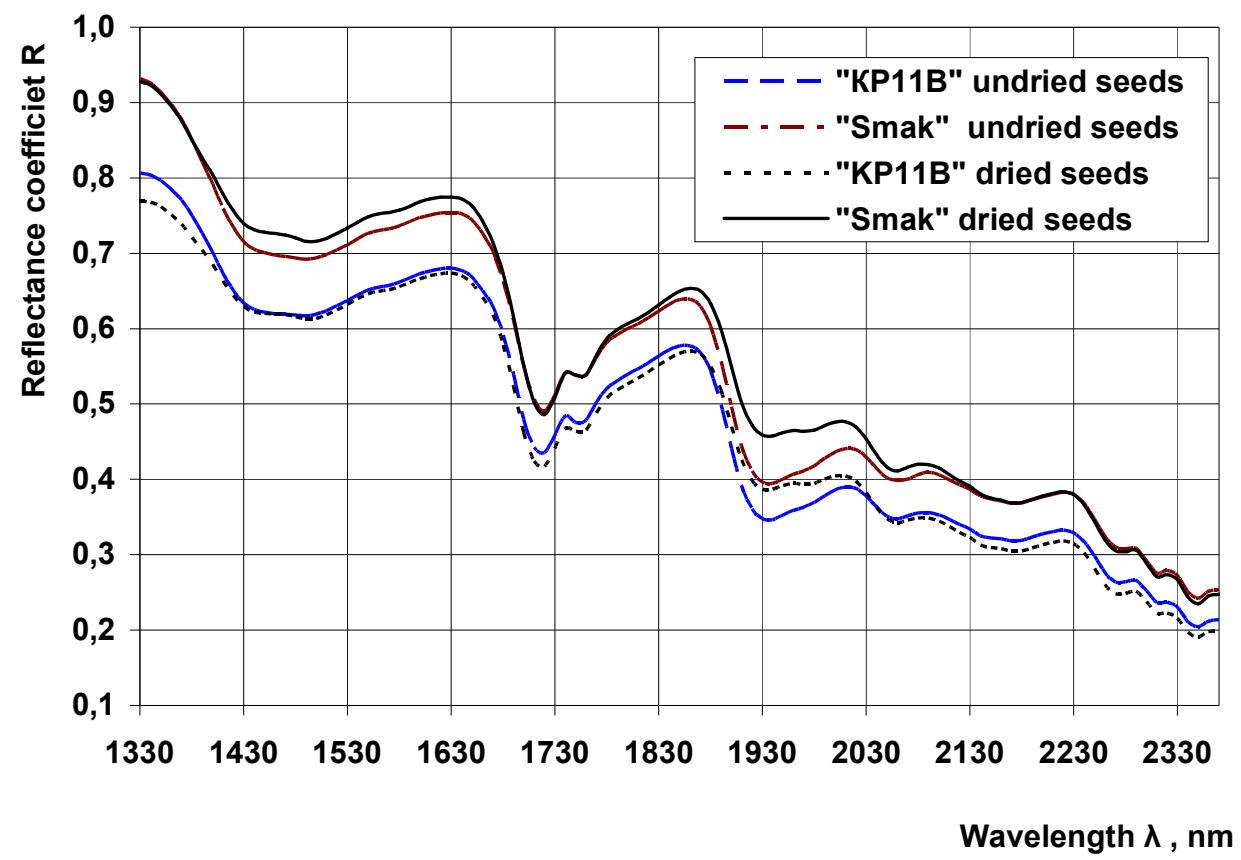

Figure 4. Diffuse reflection NIR spectra of dried samples of sunflower seed varieties "Smak" and "KP1 1B" 
When the spectra of dry and undried samples were compared, the expected differences in the intensity of diffuse reflection were found in the region 1910-1940 nm that is responsible for the water content.

\section{Development of calibration curve}

Analysis of the spectra of calibration series of sunflower seed samples enriched with free oleic acid showed the appearance of characteristic minima of the reflection intensity in the range of 2140-2160 nm (See Figure 5). The coefficient of diffuse reflection in this range increases proportionally to the growth of the mass portion of oleic acid in the sample. Calculation and construction of calibration curves and determination of linear equations on the basis of regression analysis show the dependency of the diffuse reflection coefficient on the mass portion of oleic acid at the wavelength of $2240 \mathrm{~nm}$ at a confidence level of $98 \%$ (See Figure 6).

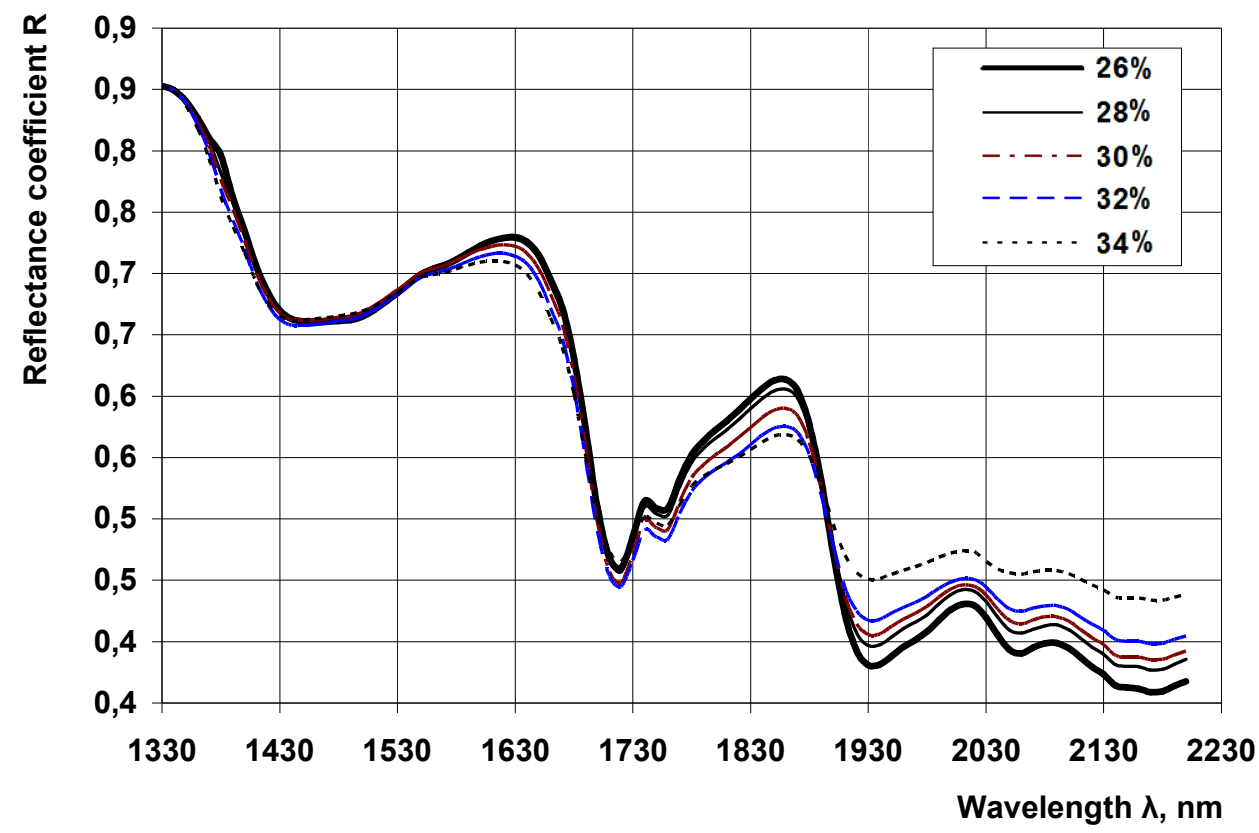

Figure 5. Diffuse reflection NIR spectra of samples of sunflower seed enriched with oleic acid 


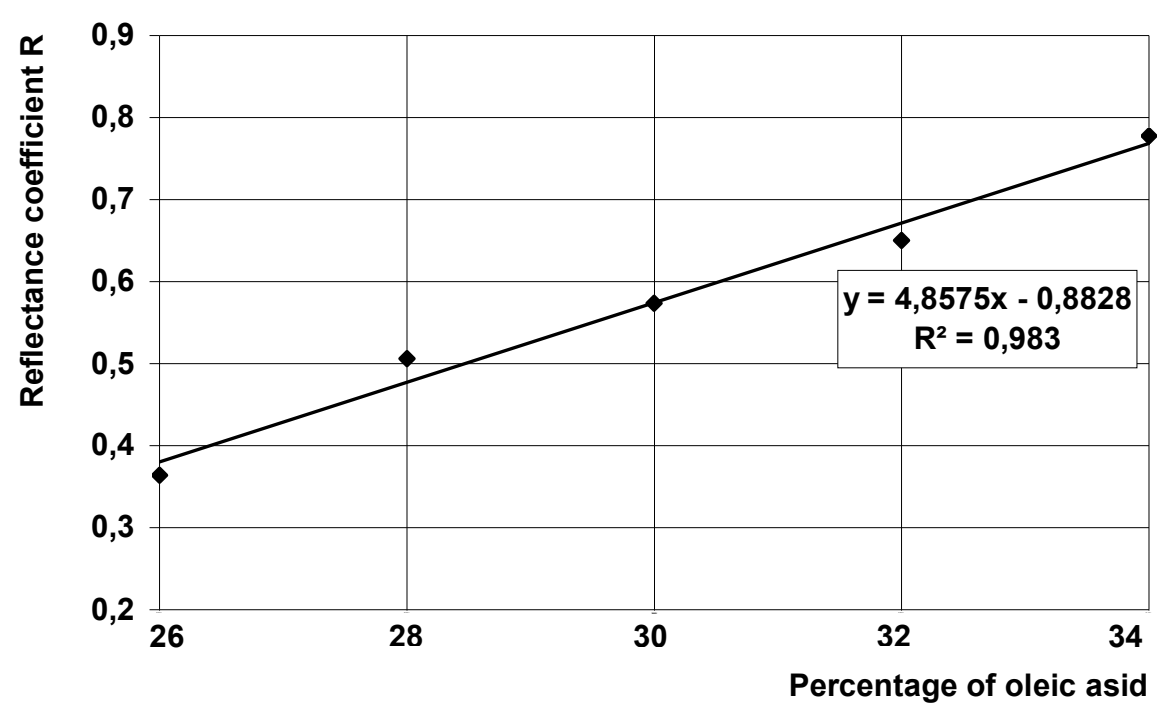

Figure 6. Calibration curve and the linear equation of dependency of the diffuse reflection coefficient on the mass portion of oleic acid at $2240 \mathrm{~nm}$

Differences in the spectra of diffuse reflection of seed samples with different amounts of free oleic acid and changes in the values of the diffuse reflection coefficient in certain regions of the spectrum are explained by the increase in the number of functional groups, that able to enter into weak interactions with both the same groups and with others [11].

Using the dependency found and knowing the value of the coefficient of diffuse reflection in the NIR range from a sample with an unknown content of oleic acid, it is possible to determine from the graph its mass portion in a given sample.

\section{Conclusions}

This non-destructive NIRS method could simplify the analysis of qualitative components, because extraction steps with organic solvents were not required and instrumental analysis was completed in a few minutes. This method for studying systems with multi component chemical composition is perspective because of the low level of light absorption in this spectral region. The method can be used to determine the wanted components over a wide range of concentrations For the analysis of numerous samples, the NIRS method can replace chemical methods and chromatographic methods such as GLC. The development of these NIR equations for individual fatty acids is only a first step though the NIRS is a practical method. The results of the comparative analysis of diffuse reflectance spectra of seed samples with husks and without husks and the same samples enriched with free oleic acid, lead to the conclusion that the method is perspective for detection of other fatty carboxylic acids in sunflower oil.

The express method of diffuse reflectance NIR spectroscopy can be considered as an alternative method for determining the quality of fat-containing materials in the processes of their storage, sorting or processing. 


\section{References}

1. Roggo Y., et al. (2007), A review of near infrared spectroscopy and chemometrics in pharmaceutical technologies, Journal of pharmaceutical and biomedical analysis, 44(3), pp. 683-700.

2. Pettersson H., Aberg L. (2003), Near infrared spectroscopy for determination of mycotoxins in cereals, Food Control, 14(4), pp. 229-232.

3. Cassoli L.D., Sartori B., Machado P.F. (2011), The use of the Fourier Transform Infrared spectroscopy to determine adulterants in raw milk, Revista Brasileira de Zootecnia, 40(11), pp. 2591-2596.

4. Blanco M., Alcala M., Bautista M. (2008), Pharmaceutical gel analysis by NIR spectroscopy: Determination of the active principle and low concentration of preservatives, European Journal of Pharmaceutical Sciences, 33(4), pp. 409-414.

5. Berardo N., et al. (2005), Rapid detection of kernel rots and mycotoxins in maize by near-infrared reflectance spectroscopy, Journal of Agricultural and Food Chemistry, 53(21), pp. 8128-8134.

6. Wang S., et al. (2009), Current status and management of chemical residues in food and ingredients in China, Trends in Food Science \& Technology, 20(9), pp. 425-434.

7. Lin H., et al.(2009), Determination of free amino acid content in Radix Pseudostellariae using near infrared (NIR) spectroscopy and different multivariate calibrations, Journal of pharmaceutical and biomedical analysis, 50(5), pp. 803-808.

8. Cen H., He Y. (2007), Theory and application of near infrared reflectance spectroscopy in determination of food quality, Trends in Food Science \& Technology, 18(2), pp. 7283.

9. Alishahi A., et al. (2010), Identification of transgenic foods using NIR spectroscopy: a review, Spectrochimica Acta Part A: Molecular and Biomolecular Spectroscopy, 75(1) pp. $1-7$.

10. Golic M., Walsh K.B. (2006), Robustness of calibration models based on near infrared spectroscopy for the in-line grading of stonefruit for total soluble solids content, Analytica chimica acta, 555(2), pp. 286-291.

11. Sánchez M.T., et al. (2010), Measurement of pesticide residues in peppers by nearinfrared reflectance spectroscopy, Pest Management Science, 66(6), pp. 580-586. 